\title{
Magnetic Resonance Imaging of Symptomatic Swimmer's Shoulder
}

\author{
Jake T Stahnke, DO*, Mari Schenk, MD and Kipling P Sharpe, MD
}

OrthoArizona, Gilbert, AZ, USA

*Corresponding author: Jake T Stahnke, DO, OrthoArizona, Gilbert, AZ, USA

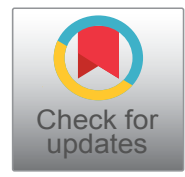

\begin{abstract}
Background: Several anterior labral lesions have been described but to our knowledge, they have not been described in the context of swimmer's shoulder.

Hypothesis: We believe a plastically deformed anterior-inferior glenoid labrum is the pathognomonic lesion in swimmer's shoulder. We believe that this can be seen but is often missed with MR arthrography.
\end{abstract}

Study design: Retrospective study.

Methods: Eleven detailed magnetic resonance imaging scans of symptomatic shoulders of swimmers who had arthroscopically demonstrated labral dysplasia were obtained between 2011 and 2014. One experienced musculoskeletal radiologist and one orthopedic surgeon interpreted each scan, specifically focusing on the anteroinferior labrum (from 3 to 6 o'clock).

Results: Nine of the eleven images (axial cuts) showed a more rounded/blunted appearance with undefined features at the anterior inferior labrum. Nine of nine images demonstrated an intact IGHL and labrum in the ABER position, three patients were unable to perform the positioning.

Conclusions: We believe the chronic repetitive micro-traumatic nature of competitive swimming leads to a more rounded/blunted appearance of the anterior inferior labrum on MR imaging. Further research may help identify these undefined features.

\section{Introduction}

One of the most common musculoskeletal complaints in competitive swimmers is shoulder pain [1,2]. In 1974, Kennedy and Hawkins described the syndrome, swimmer's shoulder, to consist of discomfort following swimming activities [3]. Since then, many researchers and clinicians have refined the definition and symptoms correlated with swimmer's shoulder; however, the mechanism of the pain and instability associated with swimmer's shoulder remains unclear [4-13]. Competi- tive swimmers practice 5-7 days a week during midseason with at least 4,000 strokes a day, sometimes significantly more. With this amount of repetition, it is important for scapular stabilizer and rotator cuff function to be well-maintained in order to avoid scapula dyskinesis; placing the shoulder at a higher risk for injury [14]. Repetitive stress on unbalanced interconnected stabilizers may lead to adaptations, including anterior inferior capsular laxity and labral pathology $[2,13,15,16]$. Unfortunately, detecting labral pathology on cross sectional imaging can be challenging as there is variability in size, thickness, and morphology of the normal labrum [1720]. Additionally, multiple normal labral variants have been described [17-20]. Several anterior labral lesions have been described but to our knowledge, they have not been described in the context of swimmer's shoulder [17,21-24]. For the purpose of this paper, we will focus on pathology related to the anterior inferior labrum.

The labrum deepens the glenoid fossa by about one third; which helps provide stability to the shoulder joint by increasing the contact area between the humeral head and the glenoid cavity [18]. The labrum is not an isolated ovoid structure but rather an interconnected periarticular fiber system with vasculature and free nerve endings reaching to the periphery. The composition of the labrum has been described as fibrocartilaginous and homogenous with the inner portion being avascular [18]. This heterogeneity gives rise to inconsistencies in signal intensity of the labrum on imaging, making it even more challenging to detect labral pathology $[18,25]$. Even though there are inconsistencies in labral signal, size, thickness, and morphology with multiple normal variants, magnetic resonance (MR) arthrography is still the best imaging modality to utilize when concerned for labral lesions $[17,18]$. 
We have found that pathology of the anterior inferior labrum in swimmers was consistently understated on preoperative MR arthrography. After re-reviewing the images, we believe a blunted and less defined anterior inferior labrum seen on axial cuts of MR arthrography is pathognomonic for plastic deformation in swimmer's shoulder.

\section{Materials and Methods}

Between January 2011-November 2014, 11 shoulder MR arthrograms (6 left and 5 right) were obtained in 9 patients with arthroscopically proved anteroinferior labroligamentous lesions. Of the 9 patients, 2 were males and 7 females; all being competitive swimmers. Age at surgery ranged from 15-28. Median age at surgery 16.5 .

After having failed formal physical therapy, MR arthrography was performed with fluoroscopic guidance. Intra-articular positioning was confirmed with a small amount of iodinated contrast material (2 cc Optiray 320 and approximately $7 \mathrm{cc}$ of a solution containing 0.15 $\mathrm{cc}$ gadolinium in $20 \mathrm{cc}$ normal saline). Using a 3.0 Tesla scanner, MR imaging of the shoulders were performed with standard sequences in three orthogonal planes following the intra-articular administration of dilute gadolinium contrast agent. Patients were positioned supine with arm in neutral position. Three patients were unable to perform ABER positioning.

During diagnostic arthroscopy, all patients had lesions at the anterior inferior labrum that was not apparent on MR arthrography. All patients underwent anterior inferior capsulorrhaphy with labral reconstruction and were able to return to competitive swimming pain free.

Over the last month, the images were re-evaluated. The surgeon and a fellowship trained musculoskeletal (MSK) radiologist were asked to independently interpret the images, specifically focusing on the anterior inferior labrum (from 3 to 6 o'clock). Coronal and axial images were used to visualize the anterior inferior labrum. The goal was to find a pattern or pathognomonic lesion at the anterior inferior labrum consistent in swimmers.

\section{Results}

(Figure 1, Figure 2, Figure 3, Figure 4, Figure 5, Figure 6, Figure 7, Figure 8, Figure 9, Figure 10 and Figure 11).
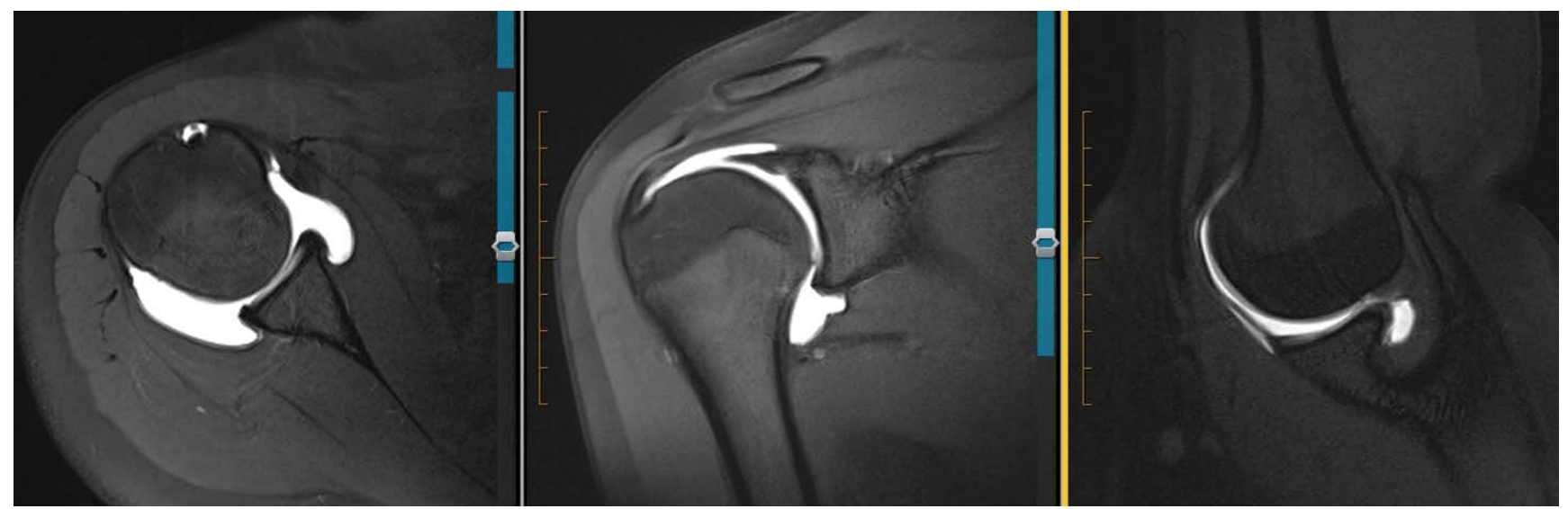

Figure 1: 16 F R MRA. From left to right: Axial T1 FS, Coronal T1 FS, and T1 FS ABER. Labrum looks blunted, rounded and undefined on axial. Nearly absent on coronal. Intact IGHL on ABER and intact labrum.

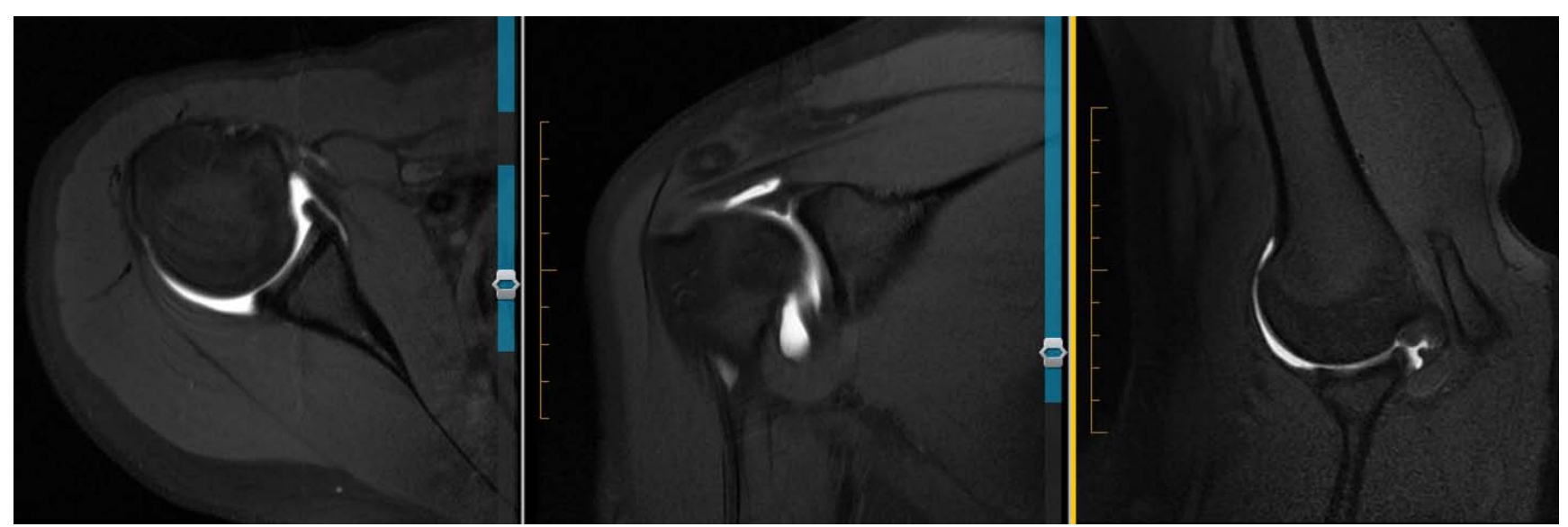

Figure 2: $16 \mathrm{~F} \mathrm{R}$ MRA. IGHL may be overlying labrum on axial view as coronal view shows thickened IGHL very well. Otherwise, labrum appears undefined. ABER view looks normal. 

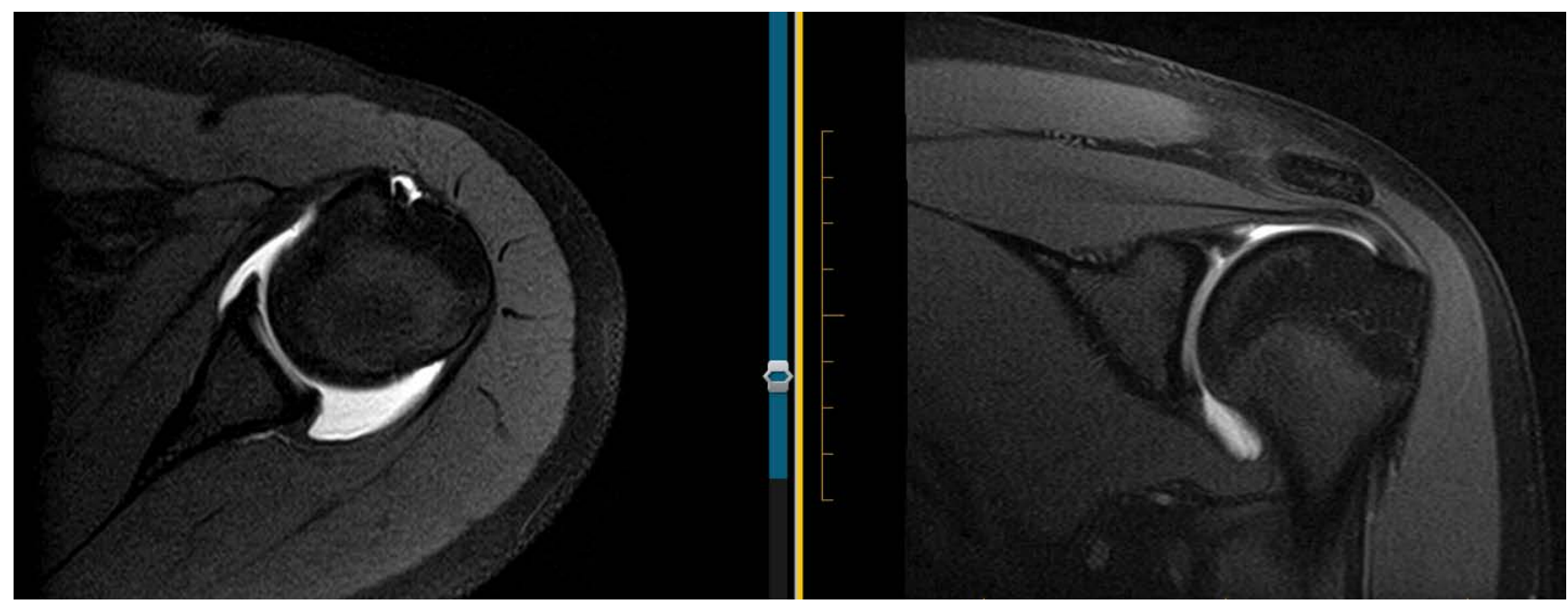

Figure 3: 16 F L MRA. Slightly more defined than others and not as blunted or rounded on axial. NO ABER views.
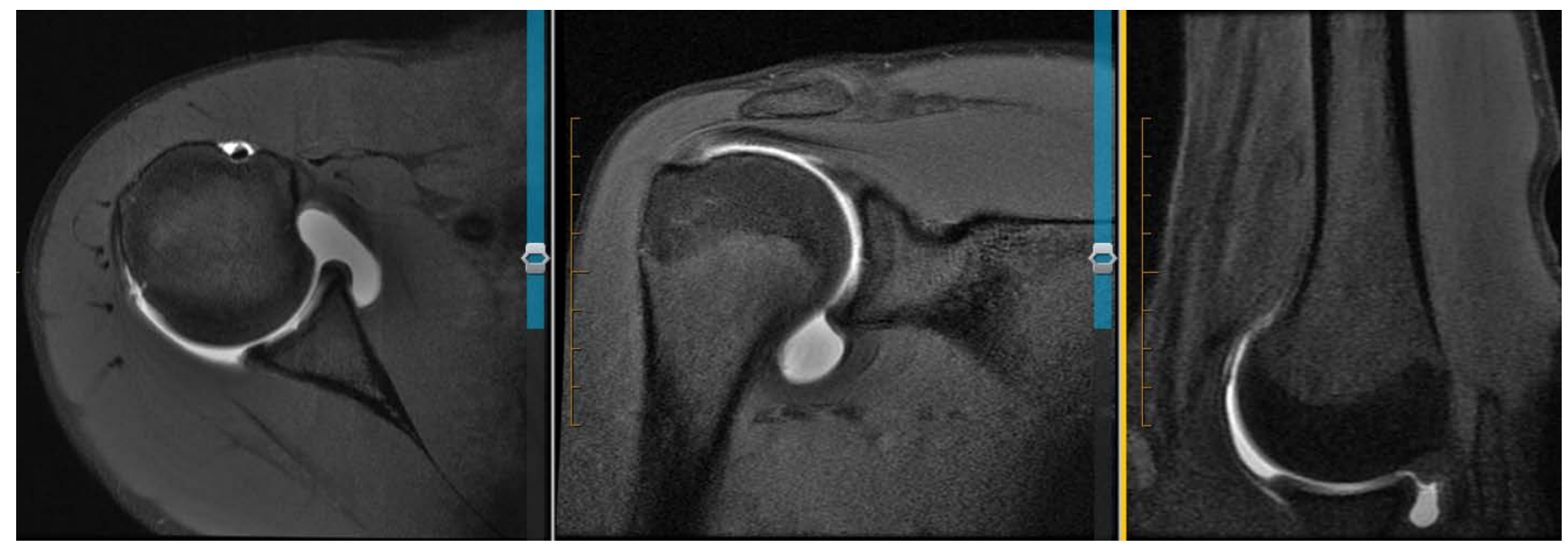

Figure 4: 16 M R MRA. Axial view shows blunted, rounded labrum. ABER view shows intact IGHL and labrum (normal).
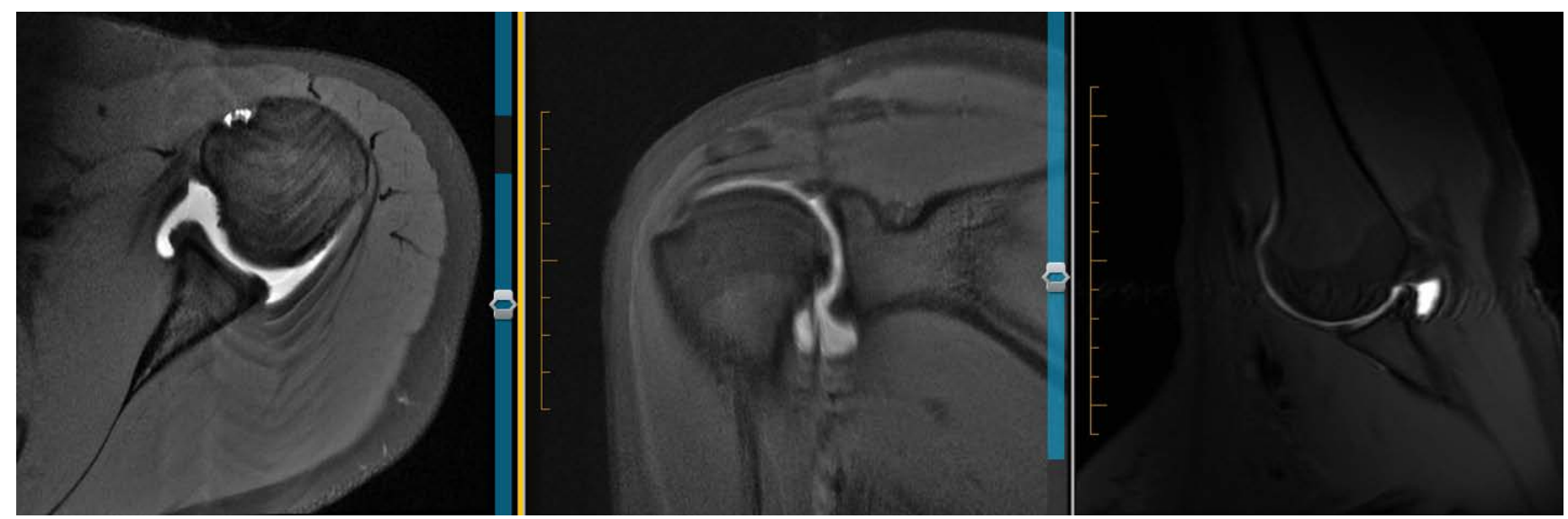

Figure 5: 17 F L MRA. Axial view shows blunted, rounded labrum. ABER view might have some undercutting of labrum, otherwise no tear of IGHL or labrum appreciated.

\section{Discussion}

The etiology of swimmer's shoulder is controversial [12]. Many have suggested that rotator cuff-related pain is the most common finding with swimmer's shoulder $[12,26]$. Some have proposed that the shoulder is at a higher risk for injury with increased fatigue $[4-6,13]$. Kennedy and Hawkins [22] hypothesized that the pain from swimmer's shoulder may be related to avascular zones around the supraspinatus and bicipital tendons [12]. Bak noted the development of swimmer's shoulder due to an unbalanced dry land and swimming training program during growth $[12,26]$. Other evidence has suggested that the primary etiology of swimmer's shoulder is tightness of the posterior capsule, as this 


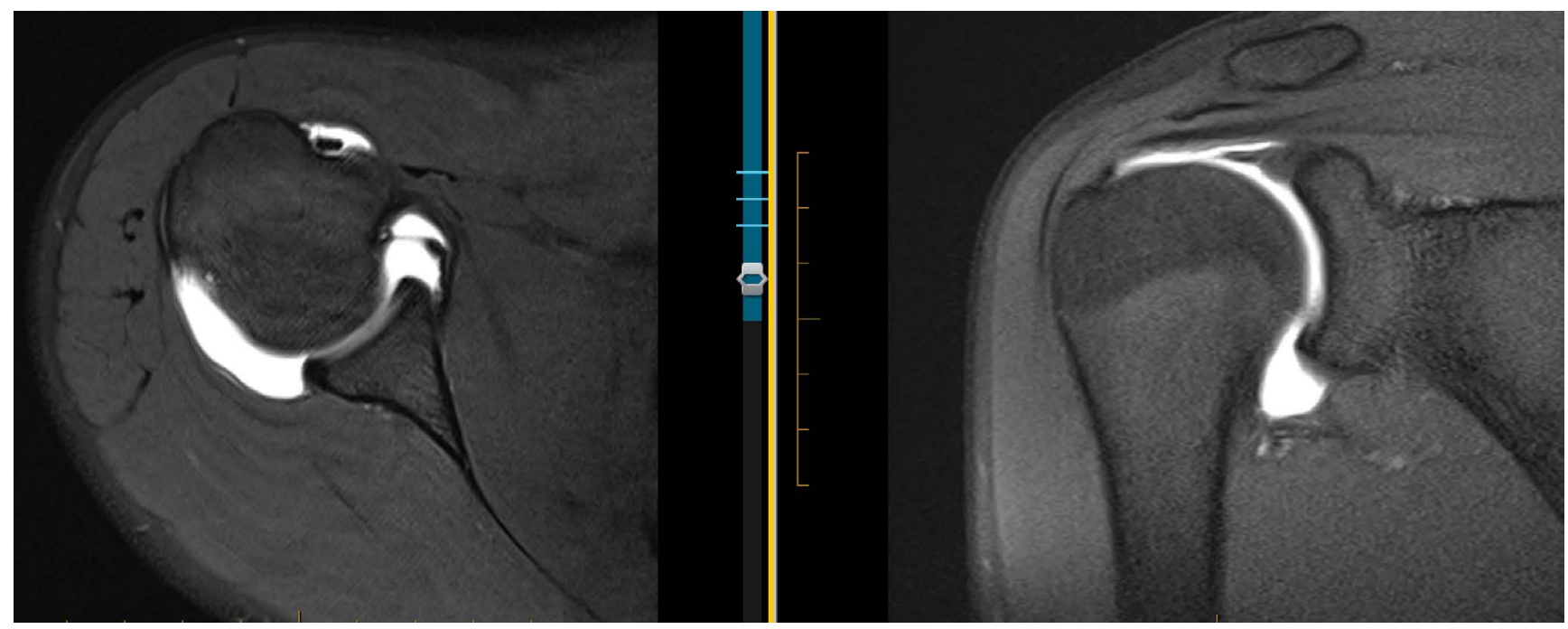

Figure 6: 17 F R MRA. Axial view shows relatively defined but still a little blunted and more rounded. NO ABER view available here.

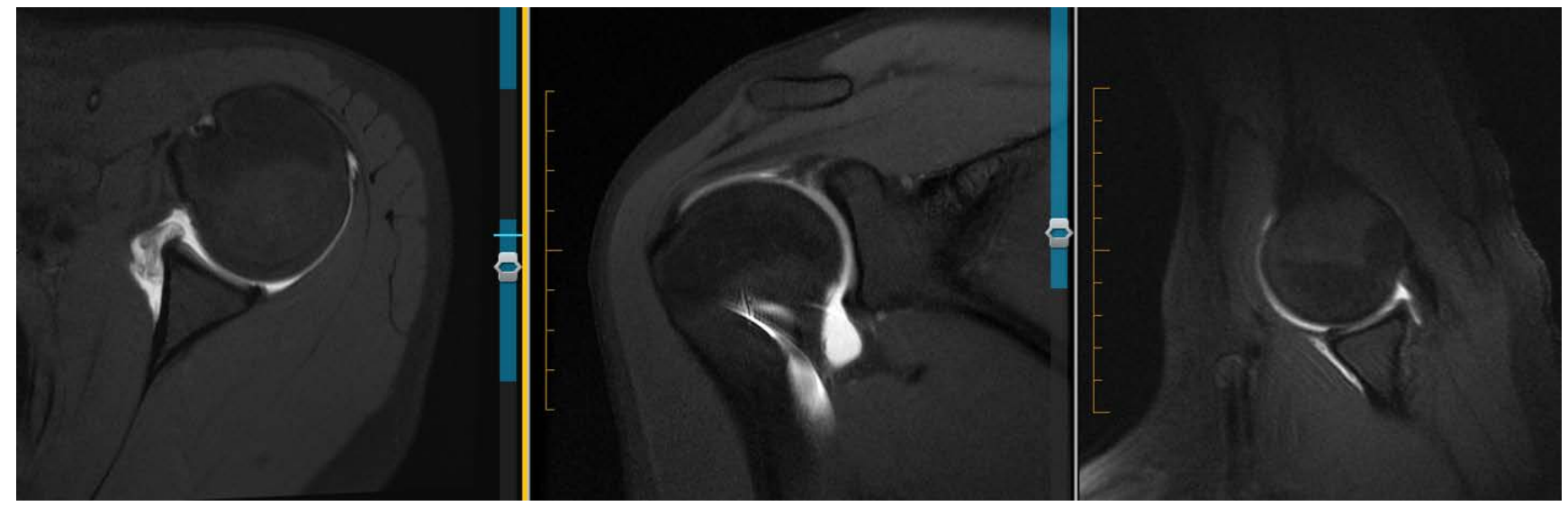

Figure 7: $17 \mathrm{~F}$ L MRA. Axial view shows blunted, rounded, and undefined anteroinferior labrum. There is some contrast dye present, likely injected into the IGHL. Which would show that it is still intact. ABER view confirms IGHL and labrum still intact.

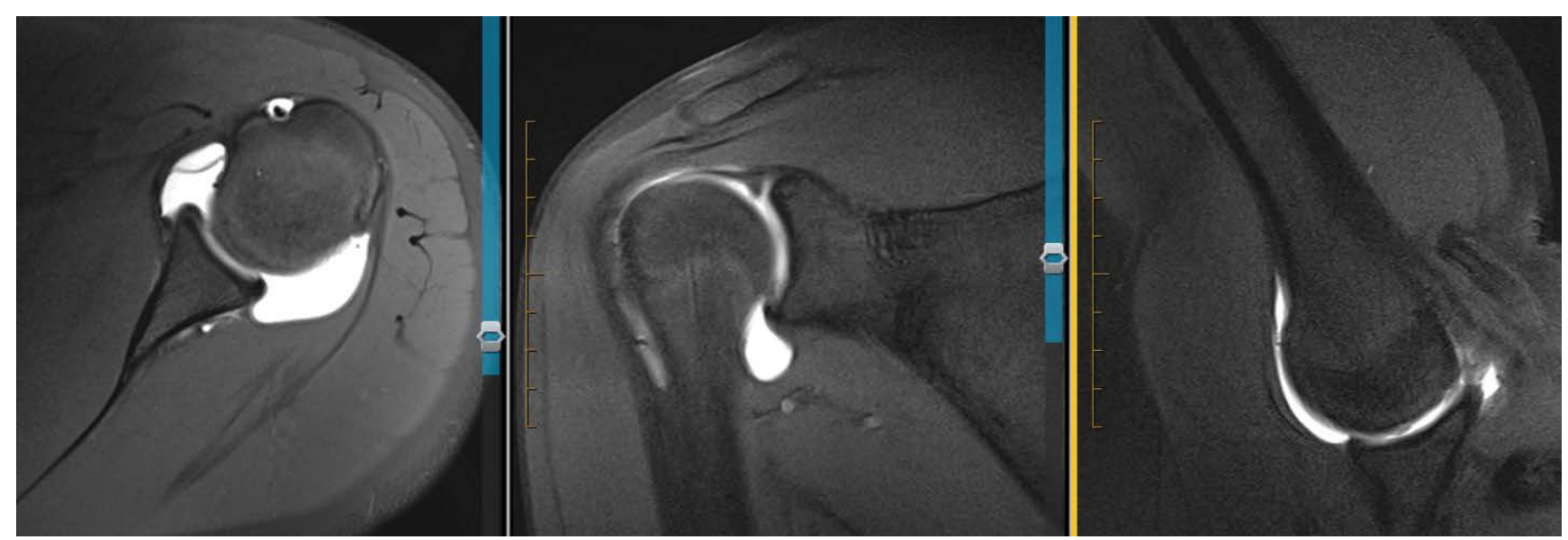

Figure 8: 17 M L MRA. Axial view shows rounding and blunting of labrum with undefined features. ABER looks normal.

has been shown to be significant in the development of internal impingement $[27,28]$. We believe scapular protraction and tightness of the posterior capsule leads to anterior capsular laxity and scapulothoracic dyskinesis. Over time, this instability along with superior translation of the humeral head during each stroke, may result in either plastic deformation of the anterior inferior labrum or a Bankart lesion, and frequently a Snyder Type II SLAP tear. Overall, we believe symptomatic swimmer's shoulder is associated with plastic deformation of the anterior inferior labrum and can be observed on MR arthrography as blunted or rounded. 

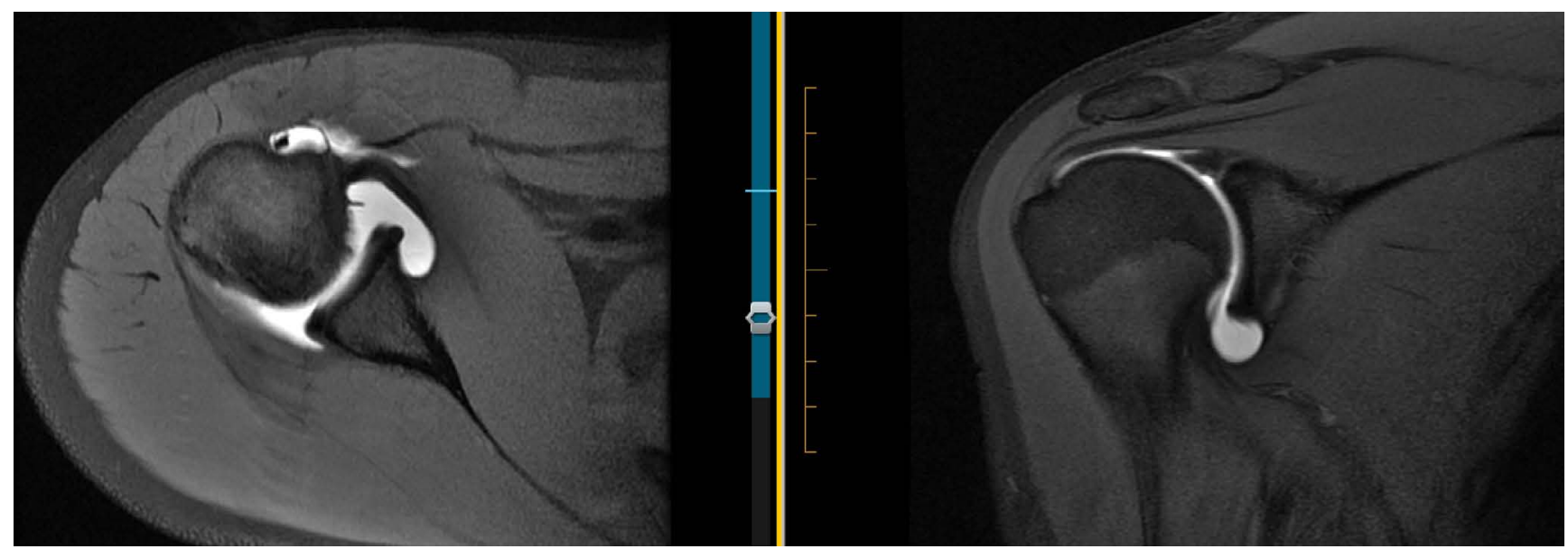

Figure 9: 15 F R MRA. Axial view shows blunted anteroinferior labrum with IGHL adjacent to it. Seems a little more defined compared with others. NO ABER views.
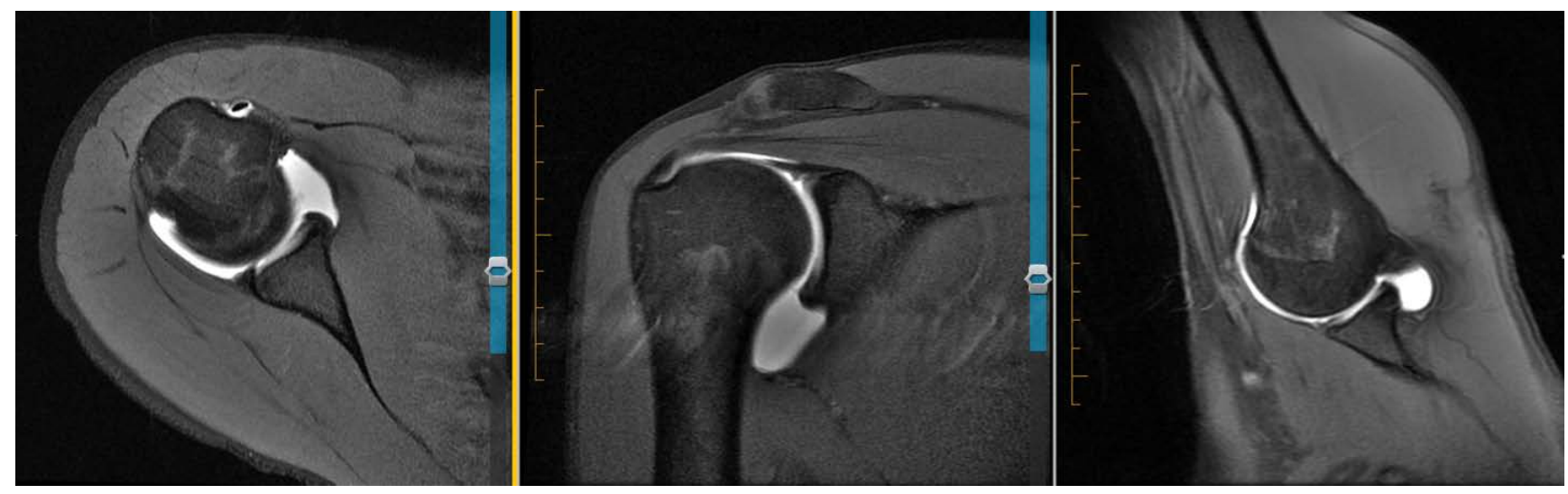

Figure 10: 28 F R MRA. Axial view shows blunting of anteroinferior labrum compared with posterior triangular appearance. ABER view appears normal. Also appears to be anterosuperior labral tear on coronal.
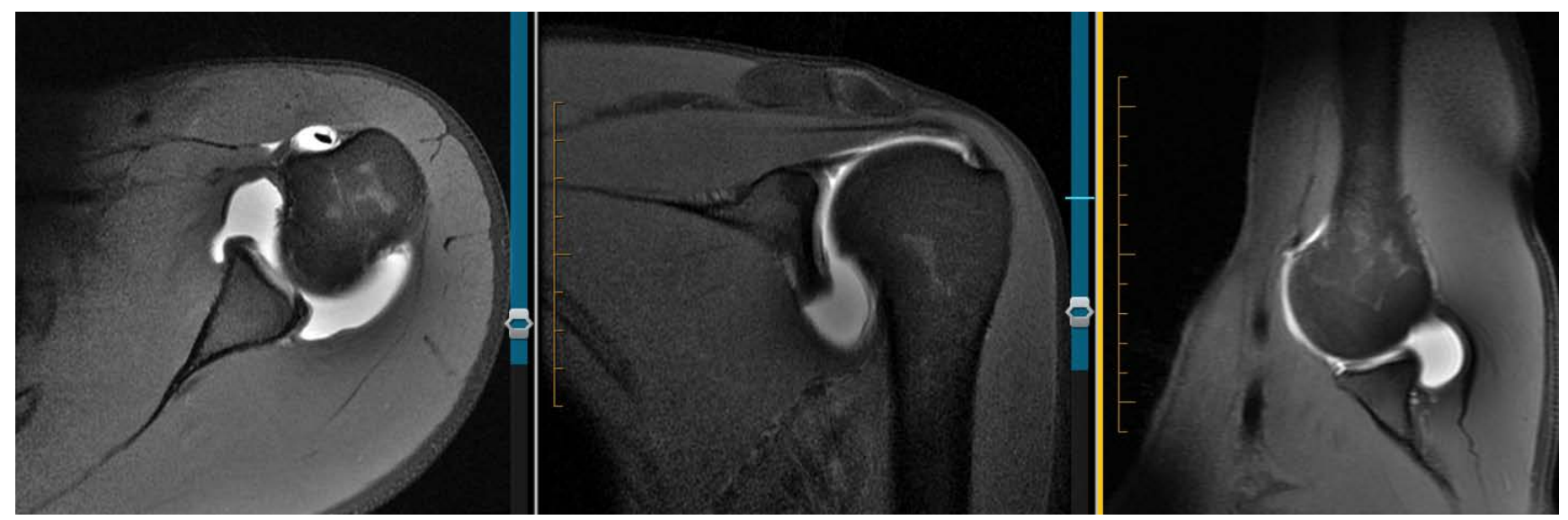

Figure 11: $28 \mathrm{~F}$ L MRA. Axial view shows blunting, rounding of anteroinferior labrum with undefined features. ABER view shows possible splitting of labrum. Anterosuperior labral tear visualized coronal. Arthroscopically -labrum and capsule were shifted medially and appeared to have a plastic deformation to them. There was no detachment or tearing of the periosteum, at most the capsule was patulous along with laxity to the IGHL but once again no tearing of any of the structures at the anteroinferior labra. Thus, no process of healing could occur (fibrosis, or scarring as seen in ALPSA).

In our study, most of the symptomatic patients initially had "normal" imaging. There appears to have been a disconnect between pathology seen on MR and pathology in surgery. We believe there needs to be a better way to identify or connect clinical symptoms of swimmer's shoulder with preoperative imaging. To our knowledge, most of the pathology at the anterior inferior labrum documented is focused around Bankart variants (ALPSA, Perthes, GLAD lesions and HAGL) [17,21$24,29,30]$. We did not appreciate labral or chondral undermining, thus, these lesions were not applicable. Furthermore, these lesions are typically used to describe 
acute trauma, whereas we believe the mechanism in swimmer's shoulder is more related to chronic repetitive microtrauma.

With at least 4,000 strokes a day, the swimmer needs a healthy balance of strong static and dynamic interconnected stabilizers otherwise the glenohumeral joint is placed at a higher risk for injury [14]. Repetitive stress on unbalanced interconnected stabilizers may lead to adaptations, including anterior inferior capsular laxity and labral pathology $[2,13,15,16]$.

In 2007, Brushoj, et al. evaluated 18 competitive swimmer's shoulders arthroscopically and found various pathologies including subacromial impingement (28\%) and labral pathology (61\%) [2,12]. In our series, 9 competitive swimmer's shoulders were treated arthroscopically, and all demonstrated labral pathology with anterior instability. All shoulders were found with lesions consistent with chronic anterior instability.

From 2011 to 2014, all images were read normal at the anterior inferior labrum yet demonstrated clear pathology arthroscopically. Looking back at the arthrograms today, we believe nine of the eleven images (axial cuts) showed a more rounded/blunted appearance with undefined features at the anterior inferior labrum.

Even though some studies have shown as high as 89 percent detection with MRA in anteroinferior lesions, these were not studies involving swimmers nor similar pathology or similar mechanisms [31]. Abduction and external rotation (ABER) of the shoulder during MR imaging puts tension on the labrum by stretching the inferior glenohumeral ligament (IGHL) [17,32]. This positioning can help peel back the anteroinferior labrum to provide better detection of anterior instability, especially Perthes lesions [17,32]. However, if the IGHL, labrum and periosteum are all still intact, then this maneuver would not aid in showing any additional pathology. In our series, three patients were unable to perform ABER. The remainder ( 6 patients, 9 images) demonstrated an intact IGHL and labrum in the ABER position.

While we believe a blunted undefined anterior inferior labrum was seen retrospectively on nine of the eleven images, the technique for looking at the anteroinferior labrum could be improved, especially in swimmers. The authors recommend adjusting the imaging settings or technique when considering MR arthrography in a competitive swimmer. We believe the best additional imaging technique is axial oblique (orthogonal to the anterior inferior labrum) with higher resolution imaging and a smaller field of view. While this adjustment might decrease signal, the scan time during this could be also decreased in order to save some of the signal. Further research and experimentation are recommended to find the best settings. Overall, the goal is to obtain a view orthogonal to the labrum, that way the chance of missing anterior inferior labral pathology in a competitive swimmer is decreased.
Unfortunately, our study is limited by a small sample size. We were limited by the number of competitive swimmers shoulder the senior author performed arthroscopy on. Additionally, we were unable to intermix good controls as the senior author did not perform surgery on asymptomatic patients, whether the MR arthrography was read as normal or not. Moreover, our study contains observer bias as the surgeon and radiologist are not blinded by the results of the arthroscopy and have previously seen the imaging. Given these biases, higher level studies are needed to better connect swimmer's shoulder, preoperative imaging, and surgical findings.

\section{Conclusion}

We believe the chronic repetitive micro-traumatic nature of competitive swimming leads to a more rounded/blunted appearance of the anterior inferior labrum on MR imaging. Further research may help identify these undefined features.

\section{Acknowledgments}

Special thanks to Mari Schenk, MD for their expertise in MRI interpretation.

\section{References}

1. Snyder SJ, Karzel RP, Del Pizzo W, RD Ferkel, MJ Friedman (1990) Slap lesions of the shoulder. Arthroscopy 6: 274-279.

2. Brushøj C, Bak K, Johannsen HV, P Faunø (2007) Swimmers' painful shoulder arthroscopic findings and return rate to sports. Scand J Med Sci Sports 17: 373-377.

3. Kennedy JC, Hawkins RJ (1974) Swimmers shoulder. Phys Sports Med 2: 34-38.

4. Pink M, Jobe FW, Perry J, J Kerrigan, A Browne, et al. (1993) The normal shoulder during the butterfly swim stroke: An electromyographic and cinematographic analysis of twelve muscles. Clin Orthop Relat Res 288: 48-59.

5. Beach ML, Whitney SL, Dickoff-Hoffman S (1992) Relationship of shoulder flexibility, strength, and endurance to shoulder pain in competitive swimmers. JOSPT 16: 262-268.

6. Allegrucci M, Whitney SL, Irrgang JJ (1994) Clinical implications of secondary impingement of the shoulder in freestyle swimmers. JOSPT 20: 307-318.

7. Pollard H, Croker D (1999) Shoulder pain is elite swimmers. ACO 8: 91-95.

8. Yanai T, Hay JG (2000) Shoulder impingement in frontcrawl swimming: II. Analysis of stroking technique. Med Sci Sports Exerc 32: 30-40.

9. Tovin BJ (2006) Prevention and treatment of swimmer's shoulder. N Am J Sports Phys Ther 1: 166-175.

10. Baril B, Bivens B, Brown L, Travis J (2008) The effects of generalized joint laxity and shoulder joint laxity on shoulder joint ROM in swimmers. Proceedings of the 4th Annual GRASP Symposium, Wichita State University.

11. Sein ML, Walton J, Linklater J, Richard Appleyard, Brent Kirkbride, et al. (2010) Shoulder pain in elite swimmers: Primarily due to swim-volume-induced supraspinatus tendinopathy. Br J Sports Med 44: 105-113. 
12. Contreras Fernández JJ, Verdugo RL, Feito MO, Rex FS (2012) Shoulder pain in swimmers. Intech 119-146.

13. Heinlein SA, Cosgarea AJ (2010) Biomechanical considerations in the competitive swimmer's shoulder. Sports Health 2: 519-525.

14. Paine R, Voight ML (2013) The role of the scapula. Int J Sports Phys Ther 8: 617-629.

15. Woertler K, Waldt S (2006) MR imaging in sports-related glenohumeral instability. Eur Radiol 16: 2622-2636.

16. Fu FH, Harner CD, Klein AH (1991) Shoulder impingement syndrome: A critical review. Clin Orthop Relat Res 269: 162-173.

17. Chloros GD, Haar PJ, Loughran TP, Hayes CW (2013) Imaging of glenoid labrum lesions. Clin Sports Med 32: 361390 .

18. De Coninck T, Ngai SS, Tafur M, Chung CB (2016) Imaging the glenoid labrum and labral tears. Radiographics. 36 : 1628-1647.

19. Smith DK, Chopp TM, Aufdemorte TB, Witkowski EG, Jones RC (1996) Sublabral recess of the superior glenoid labrum: Study of cadavers with conventional nonenhanced MR im- aging, MR arthrography, anatomic dissection, and limited histologic examination. Radiology 201: 251-256.

20. Park YH, Lee JY, Moon SH, JH Mo, BK Yang, et al. (2000) MR arthrography of the labral capsular ligamentous complex in the shoulder: Imaging variations and pitfalls. AJR Am J Roentgenol 175: 667-672.

21. Bankart AS (1923) Recurrent or habitual dislocation of the shoulder joint. Br Med J 2: 1132-1133.

22. Neviaser TJ (1993) The anterior labroligamentous periosteal sleeve avulsion lesion: A cause of anterior instability of the shoulder. Arthroscopy 9: 17-21.
23. Wischer TK, Bredella MA, Genant HK, David W Stoller, Frederic W Bost, et al. (2002) Perthes lesion (a variant of the Bankart lesion): MR imaging and MR arthrographic findings with surgical correlation. AJR Am J Roentgenol 178: 233-237.

24. Neviaser TJ (1993) The GLAD lesion: Another cause of anterior shoulder pain. Arthroscopy 9: 22-23.

25. Huber WP, Putz RV (1997) Periarticular fiber system of the shoulder joint. Arthroscopy 13: 680-691.

26. Bak K (2010) The practical management of swimmer's painful shoulder: Etiology, diagnosis, and treatment. Clin J Sport Med 20: 386-390.

27. Myers JB, Laudner KG, Pasquale MR, James P Bradley, Scott M Lephart (2006) Glenohumeral range of motion deficits and posterior shoulder tightness in throwers with pathologic internal impingement. Am J Sports Med 34: 385-391.

28. Tyler TF, Nicholas SJ, Roy T, G W Gleim (2000) Quantification of posterior capsule tightness and motion loss in patients with shoulder impingement. Am J Sports Med 28: 668-673.

29. Stoller DW (1997) MR arthrography of the glenohumeral joint. Radiol Clin North Am 35: 97-116.

30. Carlson CL (2004) The "J" Sign. Radiology 232: 725-726.

31. Waldt S, Burkart A, Imhoff AB, Melanie Bruegel, Ernst J Rummeny, et al. (2005) Anterior shoulder instability: Accuracy of MR arthrography in the classification of anteroinferior labroligamentous injuries. Radiology 237: 578-583.

32. Borrero CG, Casagranda BU, Towers JD, James P Bradley (2010) Magnetic resonance appearance of posterosuperior labral peel back during humeral abduction and external rotation. Skeletal Radiol 39: 19-26. 